\title{
A Fast-Responsive OFF-ON Near-Infrared-II Fluorescent Probe for in Vivo Detection of Hypochlorous Acid in Rheumatoid Arthritis
}

Peng $\mathrm{Wu}, \mathrm{Yu}$ Zhu, Lulu Chen, Yang Tian, and Hu Xiong*

Research Center for Analytical Sciences, Tianjin Key Laboratory of Biosensing and Molecular Recognition, College of Chemistry, Nankai University, Tianjin 300071, China.

*Correspondence should be addressed to xionghu@nankai.edu.cn

\section{Supporting Information}

\section{Table of Contents}

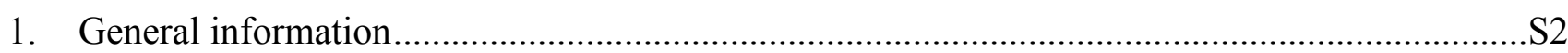

2. Supplemental figures and tables .................................................................................. 2

3. Synthesis of NIR-II fluorescent probe PTA .......................................................................... 5

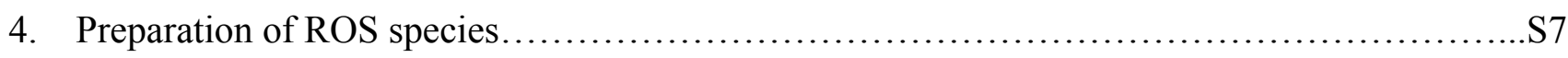

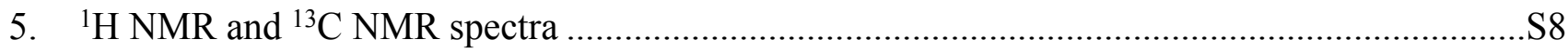

6. LC-MS data of core structures in PTA and PTA-ClO ........................................................S13

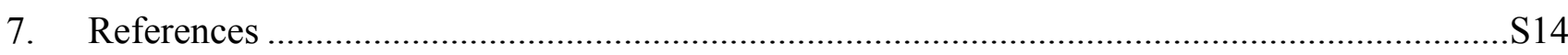




\section{General information}

\section{Chemicals and materials}

All reagents and solvents were purchased from Aladdin, Bidepharm, and J\&K and used without further purification. Dulbecco's Modified Eagle Medium (DMEM) and fetal bovine serum (FBS) were purchased from Tianjin Kaimeihong Biological Technology Co., Ltd. LysoTracker Green and DAPI were purchased from Yingwei Jieji (Shanghai) Trading Co., Ltd. Lipopolysaccharide (LPS), phorbol-12-myristate-13-acetate (PMA), and $\lambda$-carrageenan were purchased from Shanghai Macklin Biochemical Co., Ltd. $N$-acetyl- $L$-cysteine (NAC) and 4-aminobenzioc hydrazide (ABAH) were purchased from Shanghai Aladdin Biochemical Technology Co., Ltd.

\section{Instruments}

${ }^{1} \mathrm{H}$ NMR and ${ }^{13} \mathrm{C}$ NMR spectra were recorded on a Bruker $\mathrm{AM} 400 \mathrm{MHz}$ and $100 \mathrm{MHz}$ spectrometer in chloroform-d, dimethyl sulfoxide-d or methanol-d, respectively. All ${ }^{1} \mathrm{H}$ NMR signals were reported in ppm with chloroform signal at $7.26 \mathrm{ppm}$, dimethyl sulfoxide signal at 2.50 ppm, and methanol signal at $3.31 \mathrm{ppm}$ as a standard. Data for ${ }^{1} \mathrm{H}$ NMR were recorded as follows: chemical shift $(\delta, \mathrm{ppm})$, multiplicity $(\mathrm{s}=$ singlet, $\mathrm{d}=$ doublet, $\mathrm{t}=$ triplet, $\mathrm{q}=$ quartiplet, $\mathrm{m}=$ multiplet or unresolved, coupling constant(s) in $\mathrm{Hz}$, integration). All ${ }^{13} \mathrm{C}$ NMR signals were reported in ppm with the internal chloroform signal at $77.0 \mathrm{ppm}$ or dimethyl sulfoxide signal at $39.5 \mathrm{ppm}$ as a standard. Chemicals shifts are referenced to the solvent peaks and given in ppm. UV-Vis-NIR absorption spectra were recorded on a Shimadzu spectrophotometer (UV-2600 model), and fluorescence spectra were obtained by Edinburgh fluorometer (FLS1000 model). Confocal microscopy imaging was performed using a NIKON A1+ Confocal and images were analyzed using ImageJ (NIH). Whole body fluorescence imaging was performed on an NIR-II fluorescence microscopic imaging system (In-Vivo Master).

\section{Supplemental figures and tables}

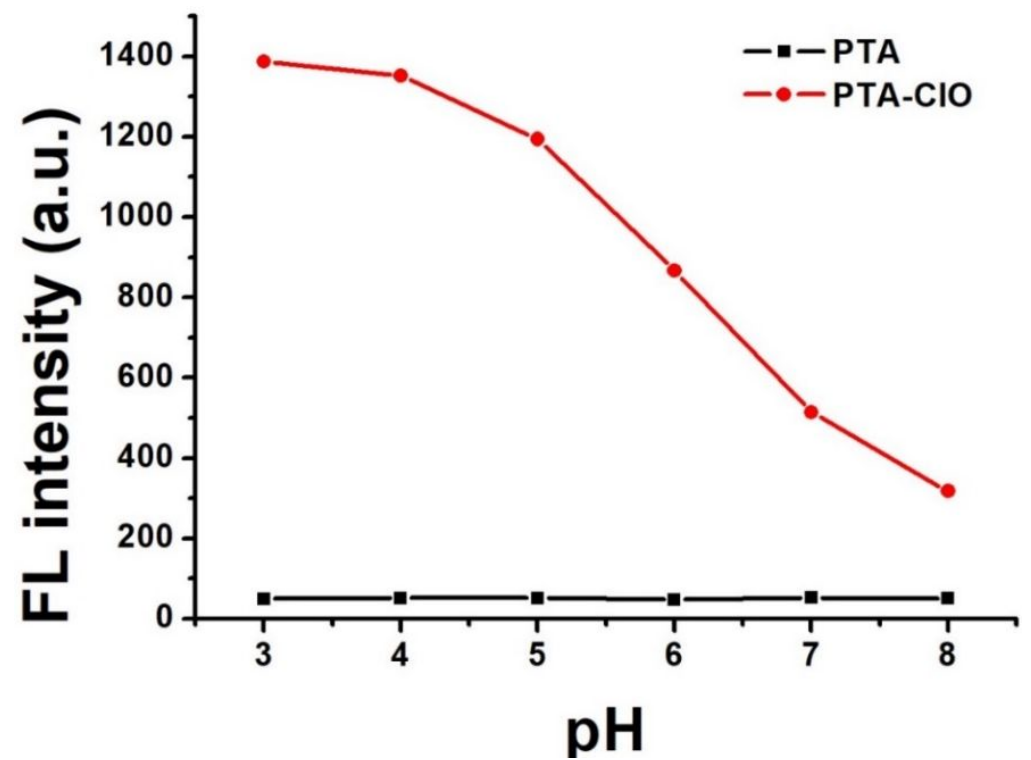

Figure S1. Fluorescence intensity of PTA $(10 \mu \mathrm{M})$ in the absence or presence of $20 \mu \mathrm{M} \mathrm{NaClO}$ in different $\mathrm{pH}$ buffer solutions. 


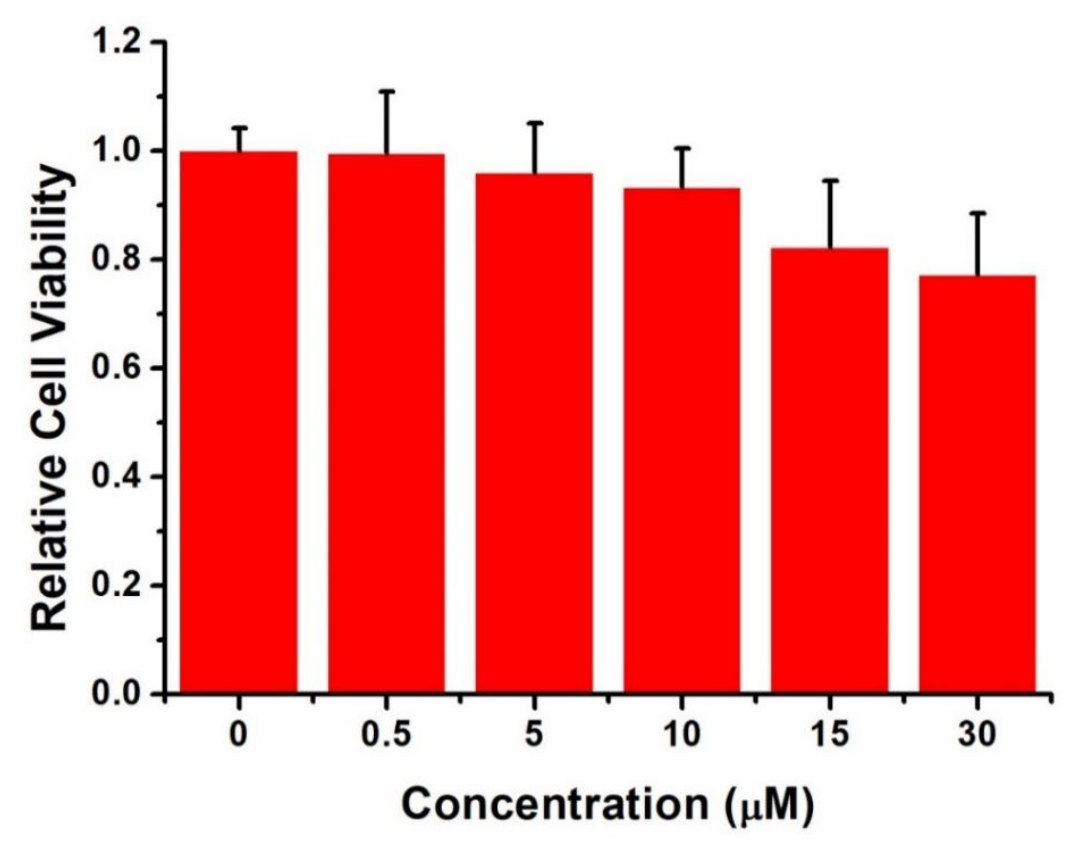

Figure S2. Cell viability of Hela cells incubated with different concentrations of PTA by CCK-8 assay $(n=3)$.

Table S1. Representative fluorescent probes based on different reaction mechanisms for imaging $\mathrm{HOCl}$.

\begin{tabular}{|c|c|c|c|}
\hline Probe & Reaction mechanism & $\lambda_{\mathrm{ab}} / \lambda_{\mathrm{em}}(\mathrm{nm})$ & In vivo applications \\
\hline Hypo-SiF $^{1}$ & $\begin{array}{l}\text { Oxidation of thioether to } \\
\text { sulfonate }\end{array}$ & $586 / 606 \mathrm{~nm}$ & -- \\
\hline MMSiR $^{2}$ & $\begin{array}{l}\text { Oxidation of thioether to } \\
\text { sulfonate }\end{array}$ & $652 / 670 \mathrm{~nm}$ & $\begin{array}{c}\text { Fluorescent imaging of } \mathrm{HOCl} \\
\text { generation in a PMA induced } \\
\text { mouse peritonitis model }\end{array}$ \\
\hline FDOCl-1 ${ }^{3}$ & Oxidation of sulfur to sulfoxide & $664 / 686 \mathrm{~nm}$ & $\begin{array}{l}\text { Fluorescent imaging } \\
\text { of } \lambda \text {-carrageenan } \\
\text { induced } \mathrm{HOCl} \\
\text { generation in mouse } \\
\text { model of arthritis }\end{array}$ \\
\hline $\mathbf{P Z}-\mathbf{P y}$ & Oxidation of sulfur to sulfoxide & $400 / 562 \mathrm{~nm}$ & $\begin{array}{l}\text { Fluorescent imaging } \\
\text { of LPS and PMA } \\
\text { induced HOCl } \\
\text { production in living } \\
\text { mice }\end{array}$ \\
\hline $\operatorname{SeCy} 7^{5}$ & $\begin{array}{l}\text { Oxidation of selenide to } \\
\text { selenoxide }\end{array}$ & $695 / 786 \mathrm{~nm}$ & $\begin{array}{l}\text { Fluorescent imaging } \\
\text { of LPS and PMA } \\
\text { induced HOCl } \\
\text { production in living } \\
\text { mice }\end{array}$ \\
\hline
\end{tabular}




\begin{tabular}{|c|c|c|c|}
\hline FO-PSe ${ }^{6}$ & $\begin{array}{c}\text { Oxidation of selenide to } \\
\text { selenoxide }\end{array}$ & $415 / 520 \mathrm{~nm}$ & -- \\
\hline RMClO-1 ${ }^{7}$ & Oxidation of Acylhydrazine & $415 / 552 \mathrm{~nm}$ & -- \\
\hline HRS1 $^{8}$ & Oxidation of Acylhydrazine & $554 / 578 \mathrm{~nm}$ & -- \\
\hline Bodipy-OX ${ }^{9}$ & $\begin{array}{c}\text { Oxidation of Hydroxylamine to } \\
\text { aldehyde }\end{array}$ & $502 / 529 \mathrm{~nm}$ & -- \\
\hline MitoClO $^{10}$ & $\begin{array}{c}\text { Oxidation of Hydroxylamine to } \\
\text { aldehyde }\end{array}$ & $505 / 529 \mathrm{~nm}$ & -- \\
\hline $\mathbf{I N C N}^{11}$ & $\begin{array}{c}\text { Oxidation of Hydrazone to } \\
\text { aldehyde }\end{array}$ & $380 / 480 \mathrm{~nm}$ & -- \\
\hline $\mathbf{P D S}^{12}$ & Oxidation of Schiff's base & $370 / 420 \mathrm{~nm}$ & -- \\
\hline PMN-TPP ${ }^{13}$ & $\begin{array}{l}\text { Cleavage of carbon- } \\
\text { carbon double bonds }\end{array}$ & $410 / 522 \mathrm{~nm}$ & $\begin{array}{l}\text { Fluorescent imaging } \\
\text { of LPS and PMA } \\
\text { induced } \mathrm{HOCl} \\
\text { production in living } \\
\text { mice } \\
\end{array}$ \\
\hline $\mathrm{ClO1}^{14}$ & $\begin{array}{l}\text { Cleavage of carbon- } \\
\text { carbon double bonds }\end{array}$ & $540 / 605 \mathrm{~nm}$ & -- \\
\hline $\mathbf{P M O P P}^{15}$ & $\begin{array}{l}\text { Oxidation of p-methoxyphenol } \\
\text { to benzoquinone }\end{array}$ & $320 / 388 \mathrm{~nm}$ & -- \\
\hline $\begin{array}{l}\text { PTA } \text { (This } \\
\text { work) }\end{array}$ & $\begin{array}{l}\text { Oxidation of sulfur to } \\
\text { sulfonium }\end{array}$ & $1020 / 936 \mathrm{~nm}$ & $\begin{array}{c}\text { Fluorescent imaging } \\
\text { of } \lambda \text {-carrageenan } \\
\text { induced } \mathrm{HOCl} \\
\text { generation in mouse } \\
\text { model of arthritis }\end{array}$ \\
\hline
\end{tabular}




\section{Synthesis of NIR-II fluorescent probe PTA}

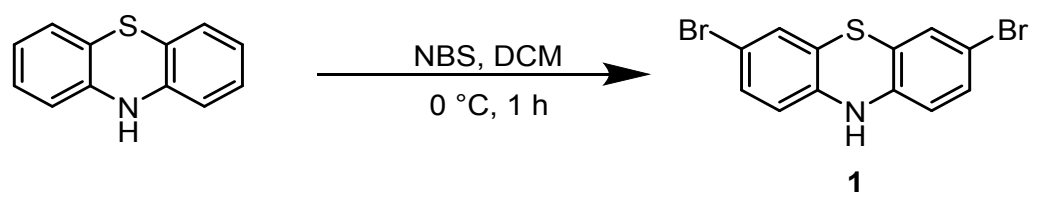

Synthesis of 1: Phenothiazine (1.99 g, $10 \mathrm{mmol})$ was dissolved in DCM (50 mL). Then, $\mathrm{N}$ bromosuccinimide (NBS, $3.92 \mathrm{~g}, 22 \mathrm{mmol}$ ) was dissolved in $45 \mathrm{~mL}$ DCM and was added dropwise over $1 \mathrm{~h}$ with an addition funnel. The reaction was stirred under an ice bath and monitored by TLC. When the reaction was finished, the mixture was poured into ice water slowly. The precipitates were collected by filtration and washed with brine, dried over anhydrous sodium sulfate, filtered, and concentrated. The crude product was purified by flash column chromatography over silica gel using EtOAc/hexanes as the eluent, affording compound 1 as white powder (2.48 g, 69\% yield). ${ }^{1} \mathrm{H}$ NMR (400 Hz, DMSO- $\left.d_{6}\right): \delta 8.83(\mathrm{~s}, 1 \mathrm{H}), 7.15-7.12(\mathrm{~m}, 4 \mathrm{H}), 6.58(\mathrm{~d}, J=7.6 \mathrm{~Hz}, 2 \mathrm{H})$.

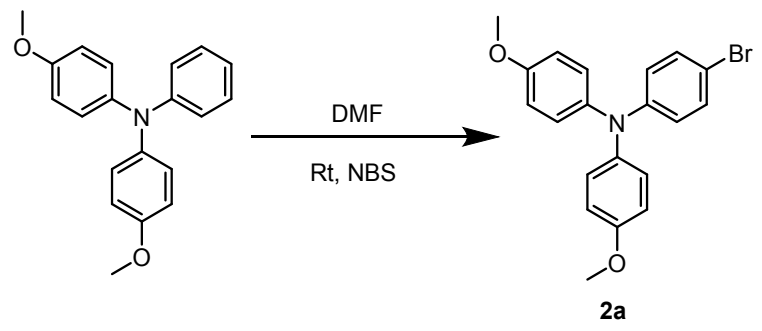

Synthesis of 2a: 4-Methoxy- $N$-(4-methoxyphenyl)- $N$-phenylaniline (6.71 g, $22 \mathrm{mmol})$ was dissolved in DMF $(100 \mathrm{~mL})$. One portion of NBS $(2.35 \mathrm{~g}, 13.20 \mathrm{mmol})$ was added at room temperature. After $30 \mathrm{~min}$, another portion of NBS (2.35 g, $13.20 \mathrm{mmol})$ was added and monitored by TLC. When the reaction was finished, the mixture was poured into ice water slowly. The precipitates were collected by filtration and washed with brine, dried over anhydrous sodium sulfate, filtered, and concentrated. The crude product was purified by flash column chromatography over silica gel using EtOAc/hexanes as the eluent, affording compound 2a as white powder (7.86 g, 93\% yield). ${ }^{1} \mathrm{H}$ NMR (400 MHz, $\left.\mathrm{CDCl}_{3}\right): \delta 7.24(\mathrm{~d}, J=9.2 \mathrm{~Hz}, 2 \mathrm{H}), 7.04$ $(\mathrm{d}, J=9.2 \mathrm{~Hz}, 4 \mathrm{H}), 6.83(\mathrm{~d}, J=9.2 \mathrm{~Hz}, 4 \mathrm{H}), 6.80(\mathrm{~d}, J=8.8 \mathrm{~Hz}, 2 \mathrm{H}), 3.80(\mathrm{~s}, 6 \mathrm{H})$.
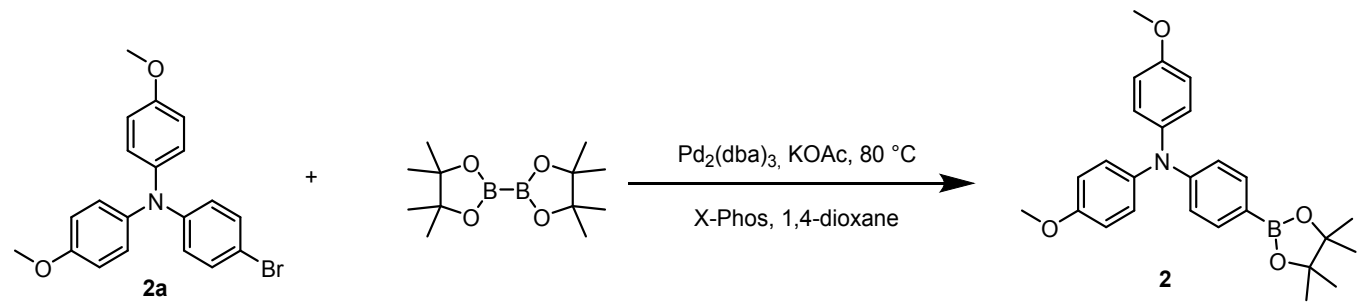

Synthesis of 2: To a $250 \mathrm{~mL}$ flask, compound 2a (7.68 g, $20 \mathrm{mmol})$, bis(pinacolato)diboron (6.10 g, $24 \mathrm{mmol}), \mathrm{Pd}_{2}(\mathrm{dba})_{3}(40 \mathrm{mg})$, X-Phos (80 mg), KOAc (1.86 g, $\left.19 \mathrm{mmol}\right)$, and dry 1,4-dioxane $(100 \mathrm{~mL})$ were added under $\mathrm{N}_{2}$ atmosphere. The mixture solution was stirred at $80{ }^{\circ} \mathrm{C}$ overnight. After removing the solvent, the crude product was purified by flash column chromatography over silica gel using EtOAc/hexanes as the eluent, affording compound 2 as white powder $(6.80 \mathrm{~g}, 79 \%$ yield). ${ }^{1} \mathrm{H}$ NMR $\left(400 \mathrm{MHz}, \mathrm{CDCl}_{3}\right): \delta 7.64(\mathrm{~d}, J=8.4 \mathrm{~Hz}, 2 \mathrm{H}), 7.09(\mathrm{~d}, J=8.8 \mathrm{~Hz}, 4 \mathrm{H}), 6.91(\mathrm{~d}$, $J=8.4 \mathrm{~Hz}, 2 \mathrm{H}), 6.85(\mathrm{~d}, J=8.8 \mathrm{~Hz}, 4 \mathrm{H}), 3.81(\mathrm{~s}, 6 \mathrm{H}), 1.35(\mathrm{~s}, 12 \mathrm{H})$. 


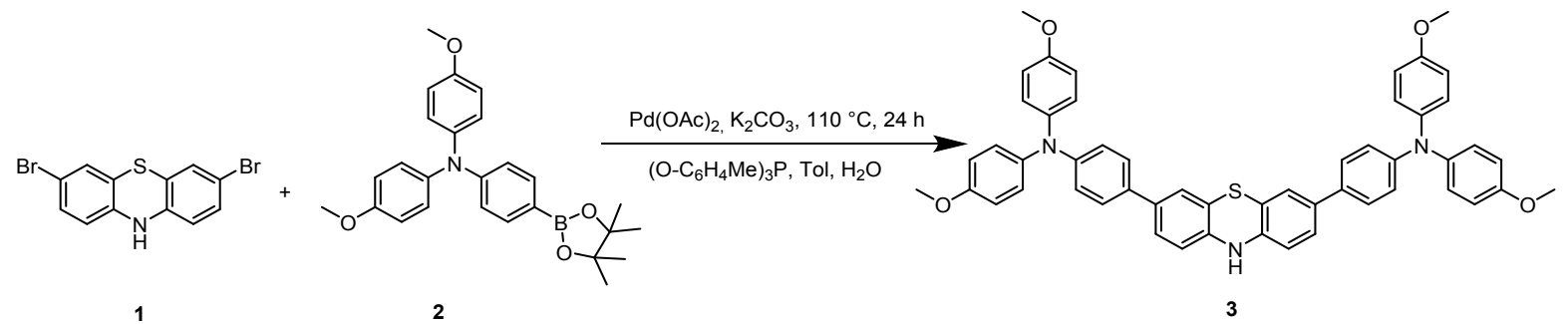

Synthesis of 3: To a $250 \mathrm{~mL}$ flask, compound 1 (2.00 g, $5.6 \mathrm{mmol})$, compound 2 (4.83 g, 11.2 $\mathrm{mmol}), \mathrm{Pd}(\mathrm{OAc})_{2}(20 \mathrm{mg}),\left(\mathrm{O}-\mathrm{C}_{6} \mathrm{H}_{4} \mathrm{Me}\right)_{3} \mathrm{P}(200 \mathrm{mg}), \mathrm{K}_{2} \mathrm{CO}_{3}(1.55 \mathrm{~g}, 11.2 \mathrm{mmol})$, dry toluene (100 $\mathrm{mL})$, and MilliQ water $(20 \mathrm{~mL})$ were added under $\mathrm{N}_{2}$ atmosphere. Then the mixture solution was stirred at $110^{\circ} \mathrm{C}$ for 24 hours. After removing the solvent, the crude product was purified by flash column chromatography over silica gel using EtOAc/hexanes as the eluent, affording compound 3 as yellow powder $\left(1.81 \mathrm{~g}, 40 \%\right.$ yield). ${ }^{1} \mathrm{H}$ NMR $\left(400 \mathrm{MHz}, \mathrm{DMSO}-d_{6}\right): \delta 8.71(\mathrm{~s}, 1 \mathrm{H}), 7.40(\mathrm{~d}, J$ $=8.8 \mathrm{~Hz}, 4 \mathrm{H}), 7.22(\mathrm{~d}, J=10.4 \mathrm{~Hz}, 2 \mathrm{H}), 7.14(\mathrm{~d}, J=2.0 \mathrm{~Hz}, 2 \mathrm{H}), 7.02(\mathrm{~d}, J=8.8 \mathrm{~Hz}, 8 \mathrm{H}), 6.91$ $(\mathrm{d}, J=9.2 \mathrm{~Hz}, 8 \mathrm{H}), 6.79(\mathrm{~d}, J=8.8 \mathrm{~Hz}, 4 \mathrm{H}), 6.71(\mathrm{~d}, J=8.0 \mathrm{~Hz}, 2 \mathrm{H}), 3.74(\mathrm{~s}, 12 \mathrm{H}) .{ }^{13} \mathrm{C}$ NMR $(100$ MHz, DMSO- $\left.d_{6}\right): \delta 155.7,147.3,140.3,140.1,133.5,130.9,126.5,126.3,125.1,123.3,119.8$, $116.7,114.9,114.7,55.2$.

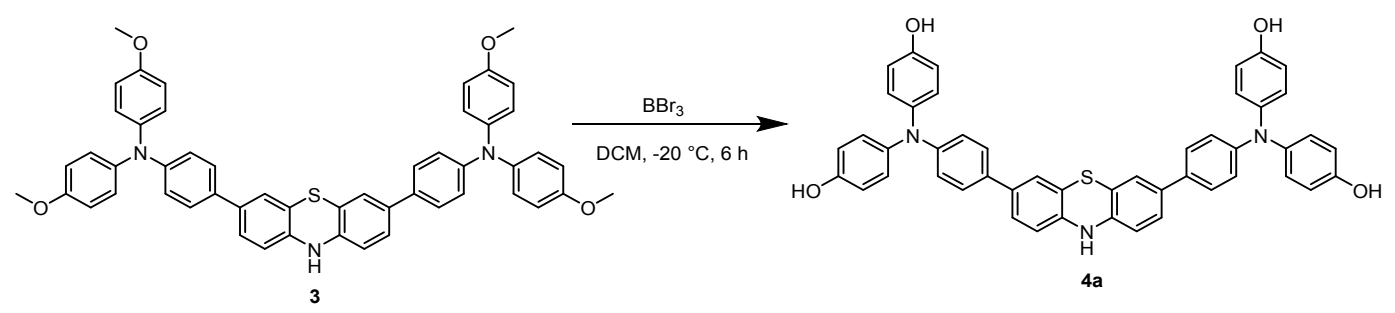

Synthesis of $4 \mathrm{a}$ : To a solution of compound $3(0.5 \mathrm{~g}, 0.62 \mathrm{mmol})$ in DCM $(20 \mathrm{~mL})$ at $-20{ }^{\circ} \mathrm{C}$, boron tribromide $\left(\mathrm{BBr}_{3}, 6.2 \mathrm{~mL}, 6.2 \mathrm{mmol}\right)$ was slowly added to the mixture under $\mathrm{N}_{2}$ atmosphere. The mixture solution was allowed to warm slowly to room temperature after addition of $\mathrm{BBr}_{3}$. When the reaction was finished, the mixture was washed with brine, dried over anhydrous sodium sulfate, filtered, and concentrated. The crude product was purified by flash column chromatography over silica gel using EtOAc/hexanes as the eluent, affording compound $\mathbf{4 a}$ as yellow oil (0.40 g, 86\% yield). ${ }^{1} \mathrm{H}$ NMR (400 MHz, DMSO- $\left.d_{6}\right): \delta 9.29(\mathrm{~s}, 4 \mathrm{H}), 8.66(\mathrm{~s}, 1 \mathrm{H}), 7.35$ $(\mathrm{d}, J=8.0 \mathrm{~Hz}, 4 \mathrm{H}), 7.20(\mathrm{~d}, J=8.0 \mathrm{~Hz}, 2 \mathrm{H}), 7.12(\mathrm{~s}, 2 \mathrm{H}), 6.92(\mathrm{~d}, J=8.8 \mathrm{~Hz}, 8 \mathrm{H}), 6.74-6.69(\mathrm{~m}$, $14 \mathrm{H}) .{ }^{13} \mathrm{C}$ NMR $\left(100 \mathrm{MHz}, \mathrm{DMSO}-d_{6}\right): \delta 154.0,147.9,140.2,138.6,133.7,129.9,127.2,126.2$, $125.0,123.3,118.5,116.7,116.2,114.7$.

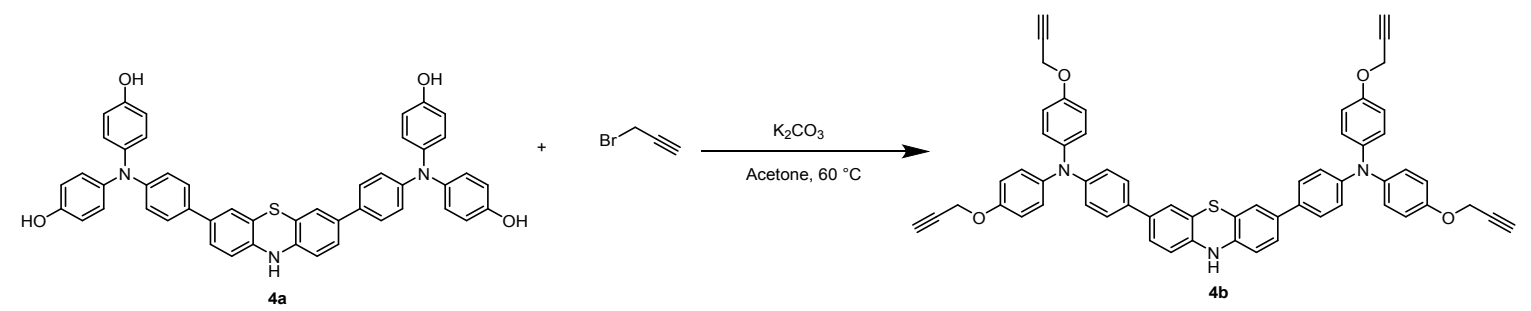

Synthesis of $4 \mathbf{b}$ : A mixture of compound $4 \mathbf{a}(0.30 \mathrm{~g}, 0.4 \mathrm{mmol}), \mathrm{K}_{2} \mathrm{CO}_{3}(0.55 \mathrm{~g}, 4 \mathrm{mmol})$, and 3-Bromo-1-propyne $(0.38 \mathrm{~g}, 3.2 \mathrm{mmol})$ in acetone $(10 \mathrm{~mL})$ was stirred at $60{ }^{\circ} \mathrm{C}$ for $24 \mathrm{~h}$ under $\mathrm{N}_{2}$ atmosphere. When the reaction was finished, the mixture was washed with brine, dried over anhydrous sodium sulfate, filtered, and concentrated. The crude product was purified by flash column chromatography over silica gel using EtOAc/hexanes as the eluent, affording compound 5 as yellow solid (0.21 g, 58\% yield). ${ }^{1} \mathrm{H}$ NMR (400 MHz, DMSO- $\left.d_{6}\right): \delta 8.70(\mathrm{~s}, 1 \mathrm{H}), 7.50-7.41(\mathrm{~m}$, 
6H), 7.24-7.16 (m, 4H), 7.04-6.95 (m, 14H), $6.82(\mathrm{~d}, J=8.4 \mathrm{~Hz}, 4 \mathrm{H}), 6.72(\mathrm{~m}, 2 \mathrm{H}), 4.77(\mathrm{~s}, 8 \mathrm{H})$, $3.57(\mathrm{~s}, 4 \mathrm{H}) .{ }^{13} \mathrm{C}$ NMR $\left(100 \mathrm{MHz}, \mathrm{DMSO}-d_{6}\right): \delta 170.3,166.9,153.6,153.5,147.1,140.7,140.6$, $131.7,131.5,128.6,126.4,126.2,120.4,115.9,79.3,78.2,55.6$.

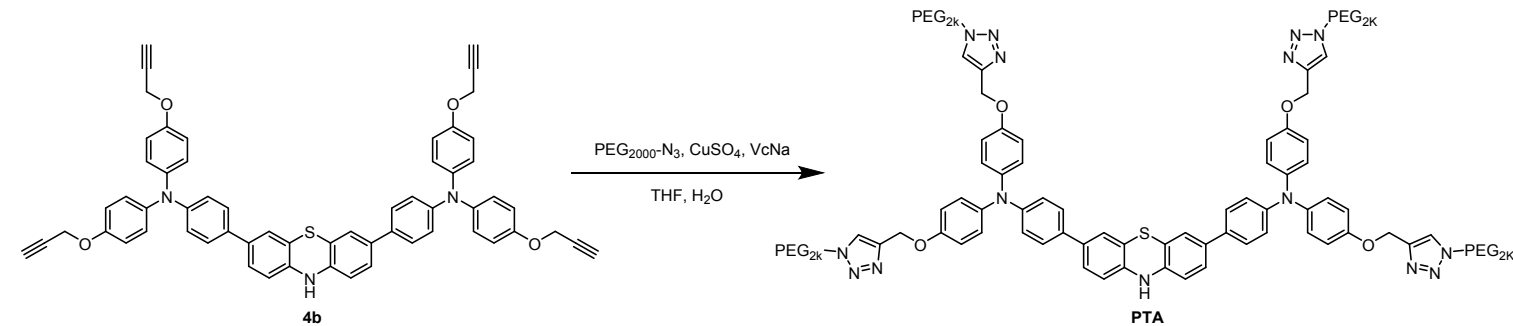

Synthesis of PTA: Compound 4b (18 mg, $0.02 \mathrm{mmol})$, methoxy-poly(ethylene glycol)-azide $\left(\mathrm{PEG}-\mathrm{N}_{3}\right.$, average $\left.\mathrm{Mn}=2000,168 \mathrm{mg}, 0.084 \mathrm{mmol}\right), \mathrm{CuSO}_{4}(1.3 \mathrm{mg}, 0.008 \mathrm{mmol})$, and sodium ascorbate (VcNa, $15.8 \mathrm{mg}, 0.08 \mathrm{mmol}$ ) were added to a $10 \mathrm{~mL}$ Schlenk flask. The vessel was evacuated and back-filled with $\mathrm{N}_{2}$. Then $4 \mathrm{~mL}$ THF (degassed) was added via a syringe, followed by $1 \mathrm{~mL}$ DI water (degassed). The reaction mixture stirred overnight at room temperature. The residue was dialyzed in DI water for $6 \mathrm{~h}$ and lyophilized to obtain a yellow powder. The collected product was then purified by column chromatography through a Sephadex LH-20 resin using $\mathrm{MeOH}$ as the eluent, affording PTA as yellow solid (150 mg, 84\% yield). ${ }^{1} \mathrm{H}$ NMR (400 MHz, Methanol- $\left.d_{4}\right): \delta 8.16(\mathrm{~s}, 4 \mathrm{H}), 7.38-7.36(\mathrm{~m}, 6 \mathrm{H}), 7.22-7.14(\mathrm{~m}, 4 \mathrm{H}), 7.04-6.97(\mathrm{~m}, 14 \mathrm{H}), 6.91(\mathrm{~d}$, $J=8.4 \mathrm{~Hz}, 4 \mathrm{H}), 6.70(\mathrm{~d}, J=8.4 \mathrm{~Hz}, 2 \mathrm{H}), 5.16(\mathrm{~s}, 8 \mathrm{H}), 4.61(\mathrm{t}, J=4.8 \mathrm{~Hz}, 8 \mathrm{H}), 3.90(\mathrm{t}, J=4.8 \mathrm{~Hz}$, $8 \mathrm{H}), 3.74-3.49(\mathrm{~m}, 696 \mathrm{H}), 3.35(\mathrm{~s}, 12 \mathrm{H})$.

\section{Preparation of ROS species}

$\mathrm{ClO}^{-}$solution $(1 \mathrm{mM})$ was freshly prepared by dissolving $\mathrm{NaClO}$ into deionized water. The $\mathrm{NO}$ solution was prepared by adding SNP (Sodium nitroprusside (III) dihydrate) into degassed deionizer water under $\mathrm{N}_{2}$ atmosphere, and then stirred for 40 min at $25{ }^{\circ} \mathrm{C} . \mathrm{H}_{2} \mathrm{O}_{2}$ and ${ }^{\mathrm{t}} \mathrm{BuOOH}$ solutions $(1 \mathrm{mM})$ were obtained by dilution of commercial $30 \% \mathrm{H}_{2} \mathrm{O}_{2}$ and $70 \%{ }^{\mathrm{t}} \mathrm{BuOOH}$ with deionized water. $\mathrm{ONOO}^{-}$solution was generated as follows: To the vigorously stirred solution of $\mathrm{NaNO}_{2}(0.6 \mathrm{M})$ and $\mathrm{H}_{2} \mathrm{O}_{2}(0.7 \mathrm{M})$, a solution of $\mathrm{HClO}(0.6 \mathrm{M})$ was added, and then $\mathrm{NaOH}(1 \mathrm{M})$ was quickly added to make the solution alkaline. The solution was passed through a short column of manganese dioxide to eliminate excess hydrogen peroxide. The concentration of $\mathrm{ONOO}^{-}$was determined by measuring the absorption of the solution at $302 \mathrm{~nm}$ with an extinction coefficient of $1670 \mathrm{M}^{-1} \mathrm{~cm}^{-1}$. $\cdot \mathrm{OH}(1 \mathrm{mM})$ was generated in situ via Fenton reaction between $\mathrm{FeCl}_{2}$ and $\mathrm{H}_{2} \mathrm{O}_{2}$ quantitively, and $\mathrm{FeCl}_{2}$ concentration represented $\bullet \mathrm{OH}$ concentration. ${ }^{1} \mathrm{O}_{2}$ was generated by the reaction between $\mathrm{NaClO}(1 \mathrm{mM})$ and $\mathrm{H}_{2} \mathrm{O}_{2}(1 \mathrm{mM})$. 


\section{5. ${ }^{1} \mathrm{H}$ NMR and ${ }^{13} \mathrm{C}$ NMR spectra}
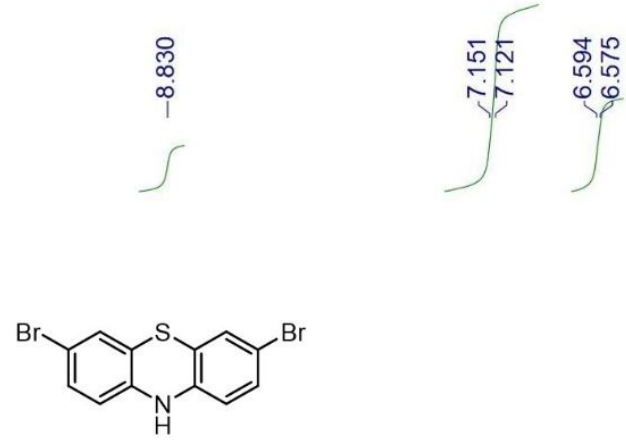

1

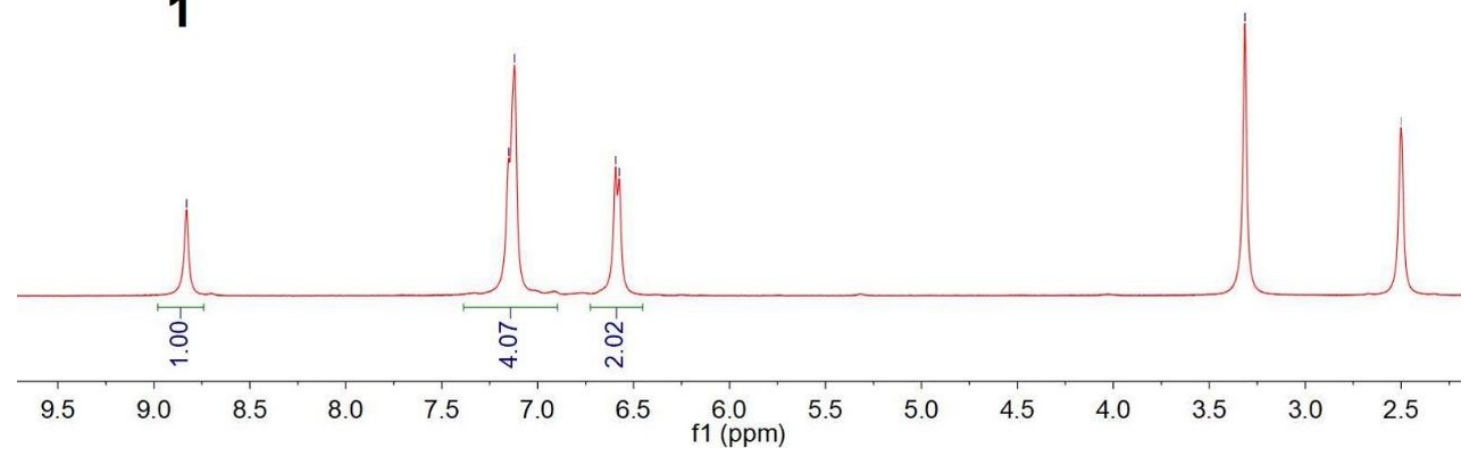

整.1.10.fid

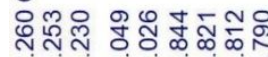

Nîn

तर पर
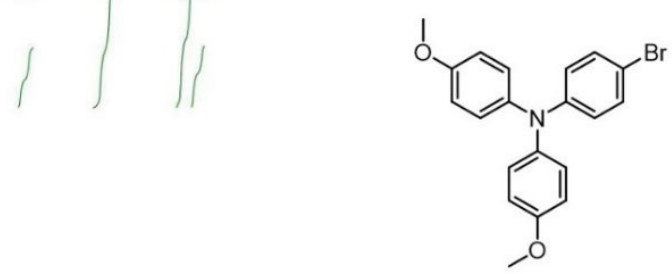

$2 a$

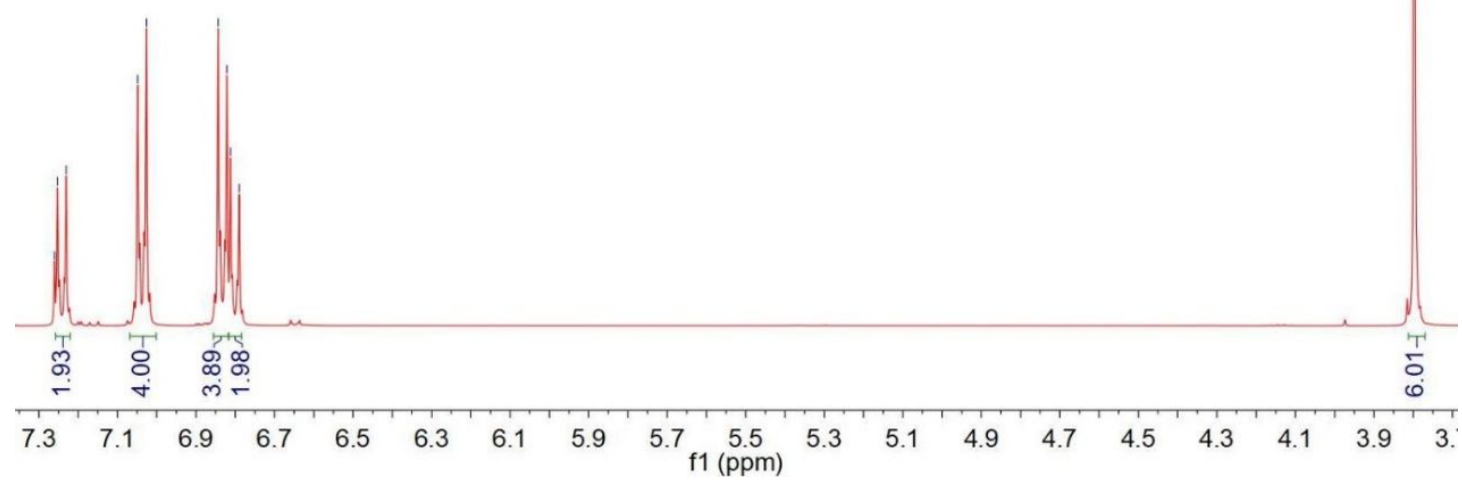




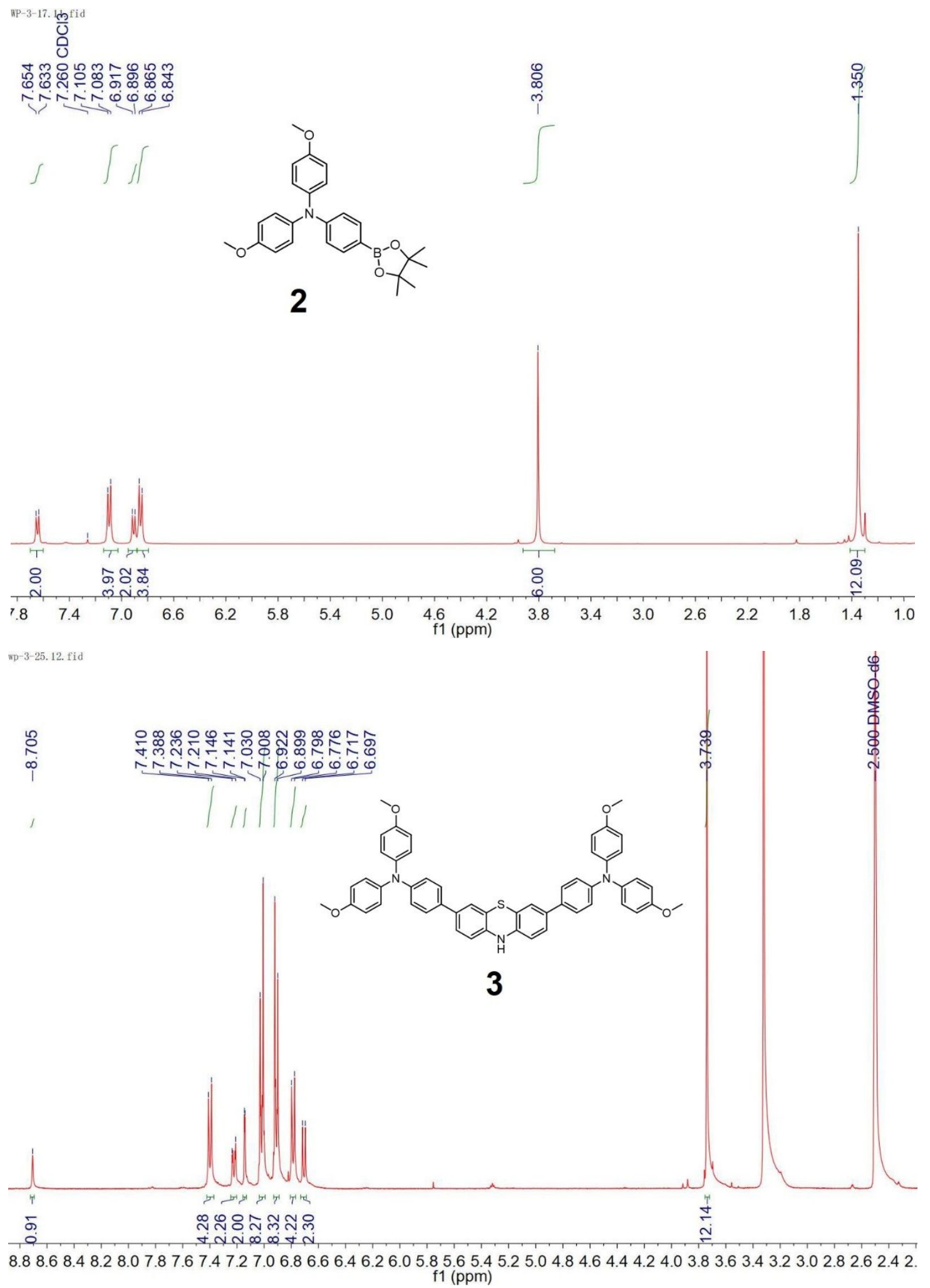




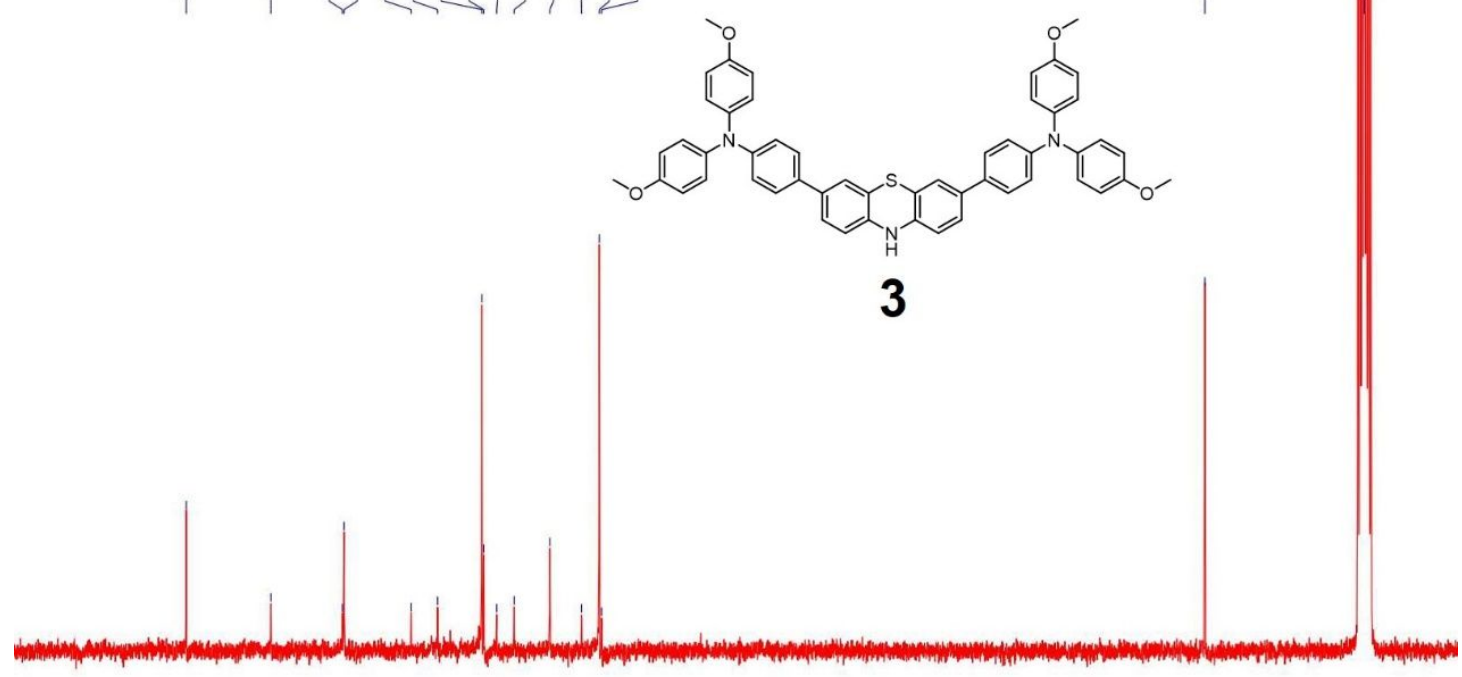

$\left.170165160155150145140135130125120115110 \begin{array}{c}105100 \\ \mathrm{f} 1(\mathrm{ppm})\end{array}\right)$
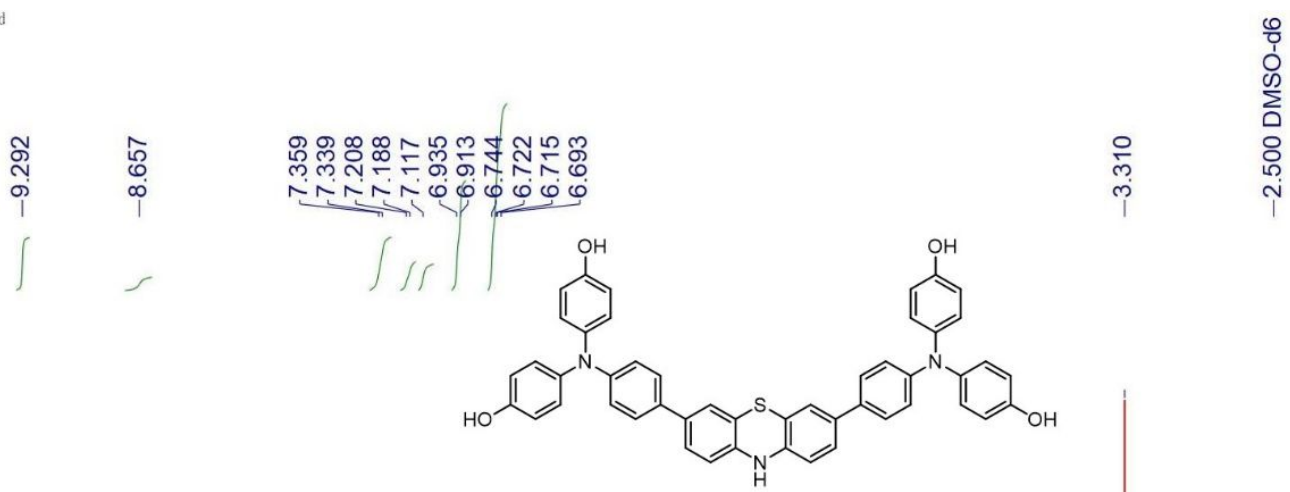

$4 a$

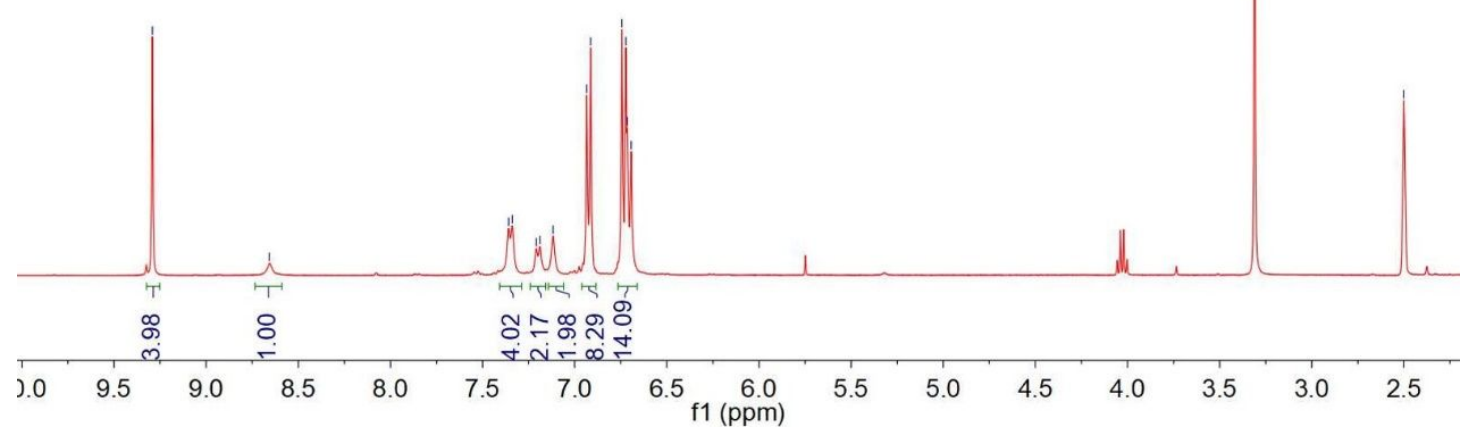


¿

i

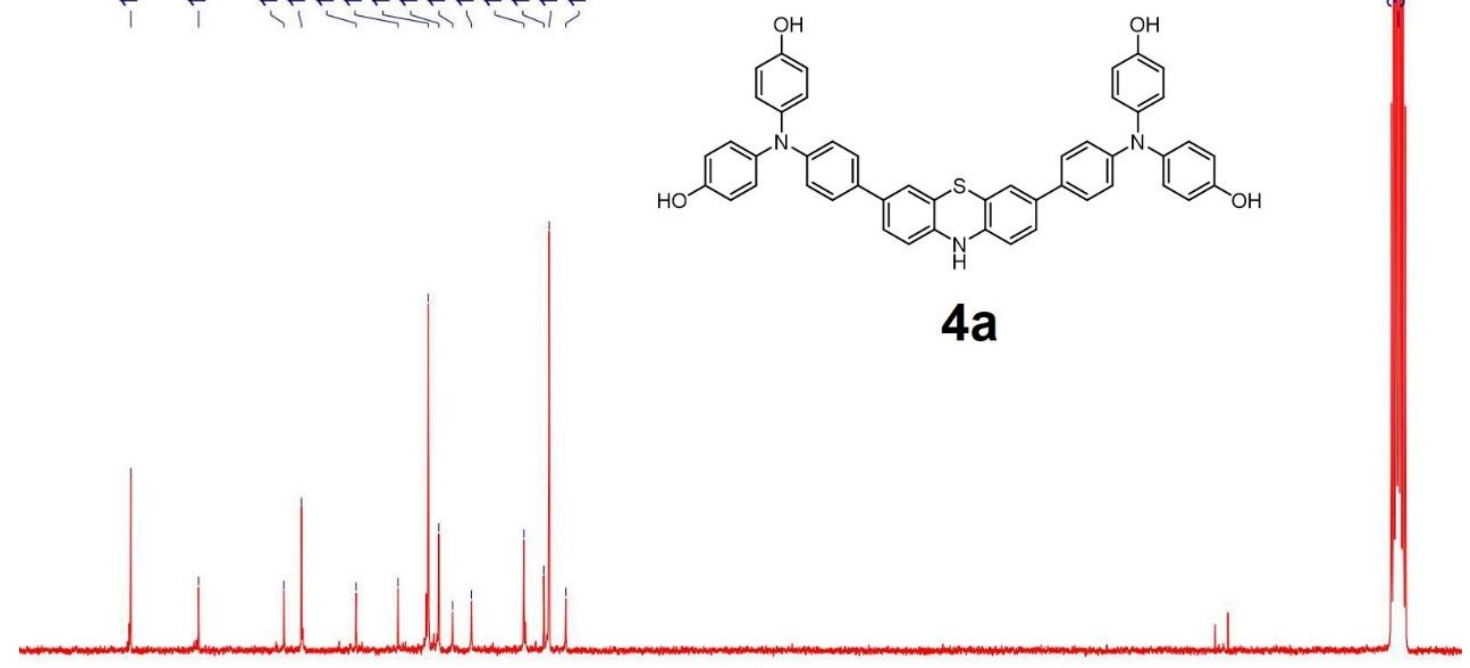

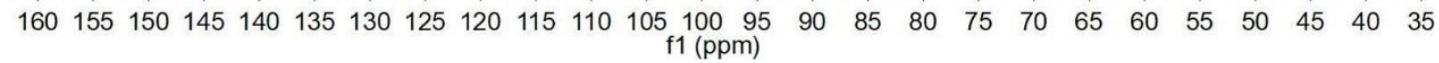
wp-3-62. 13. fid

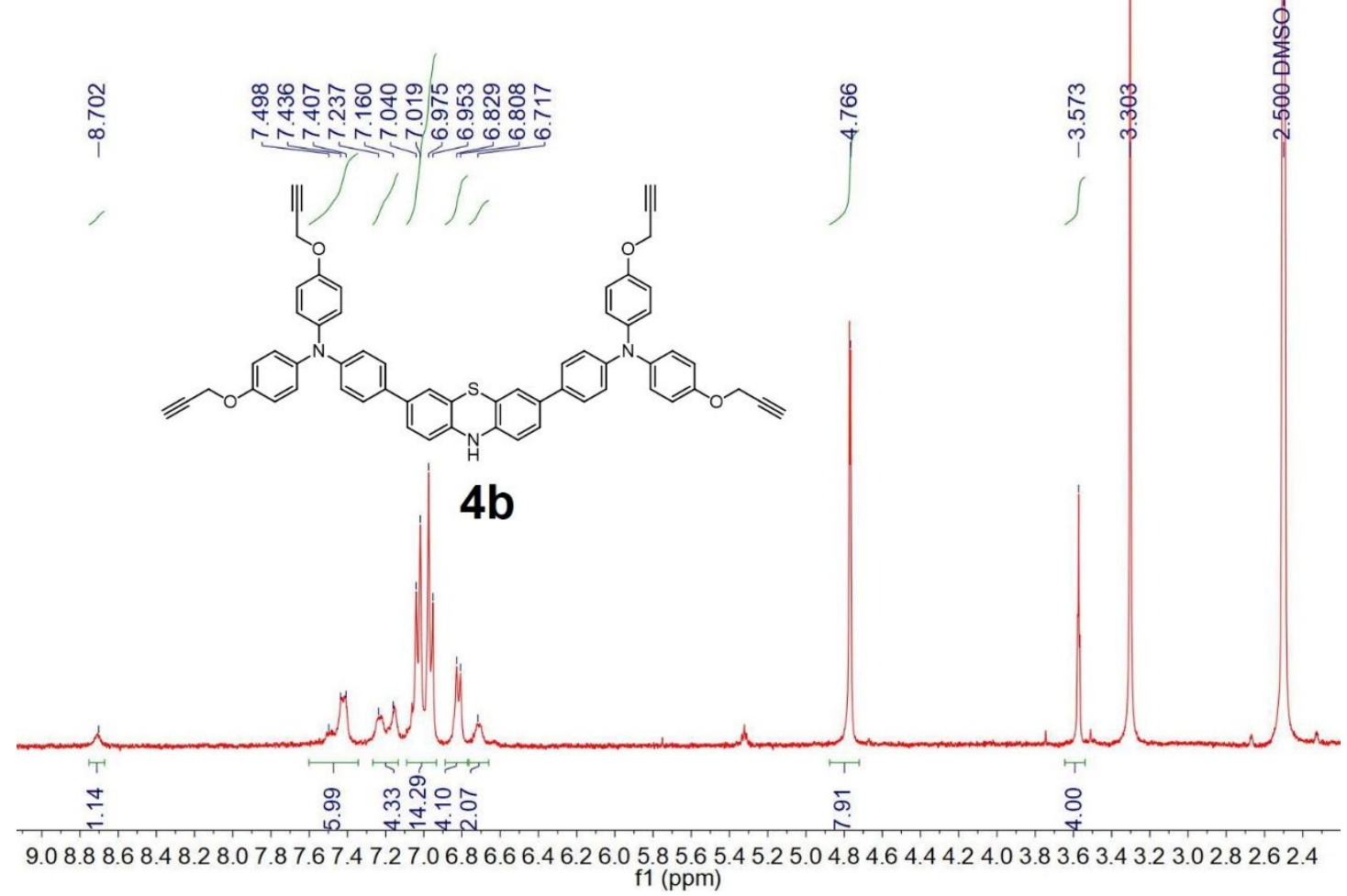




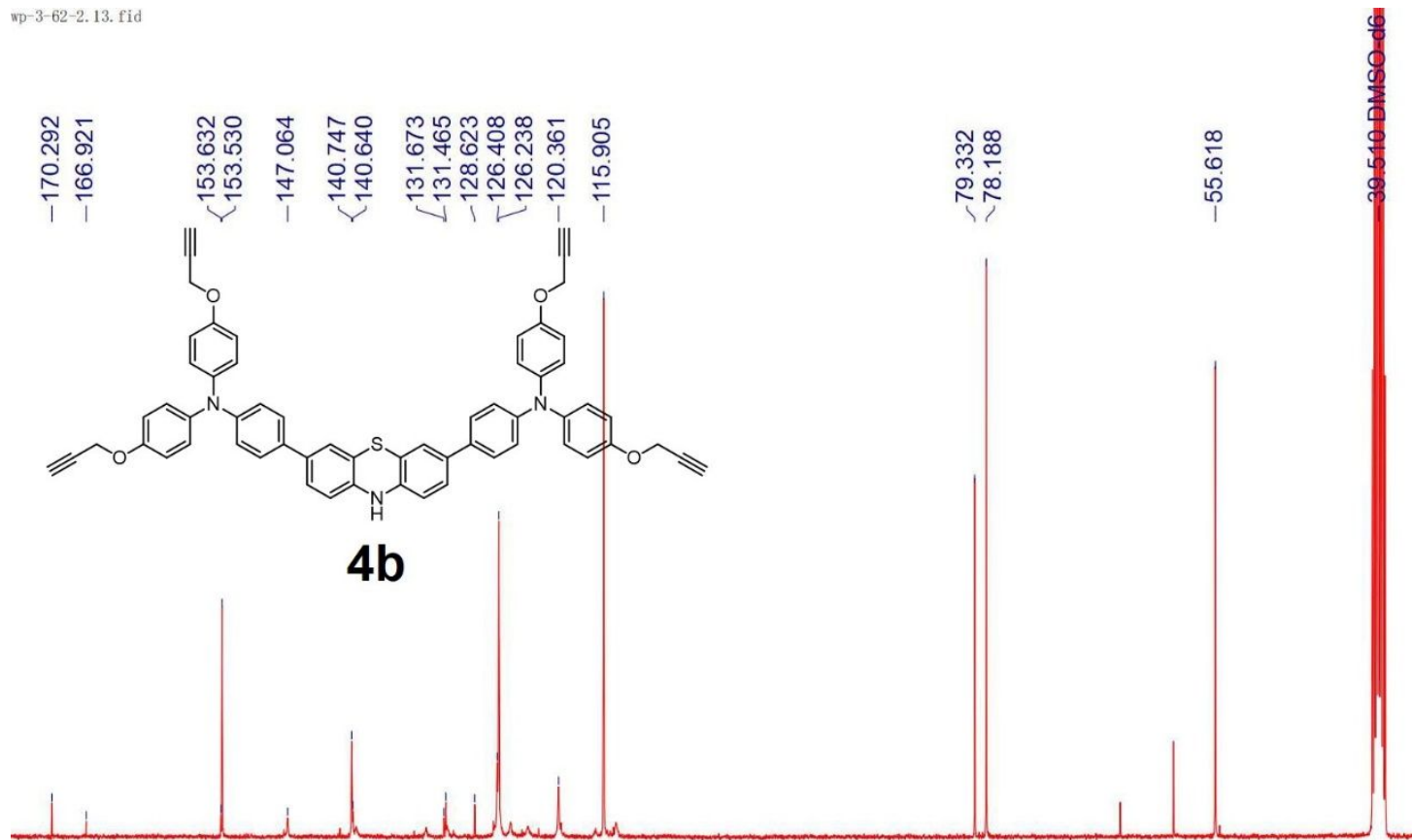

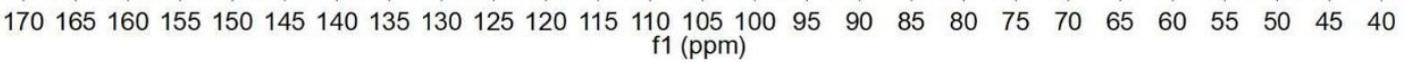
wp-3-63. 15. fid

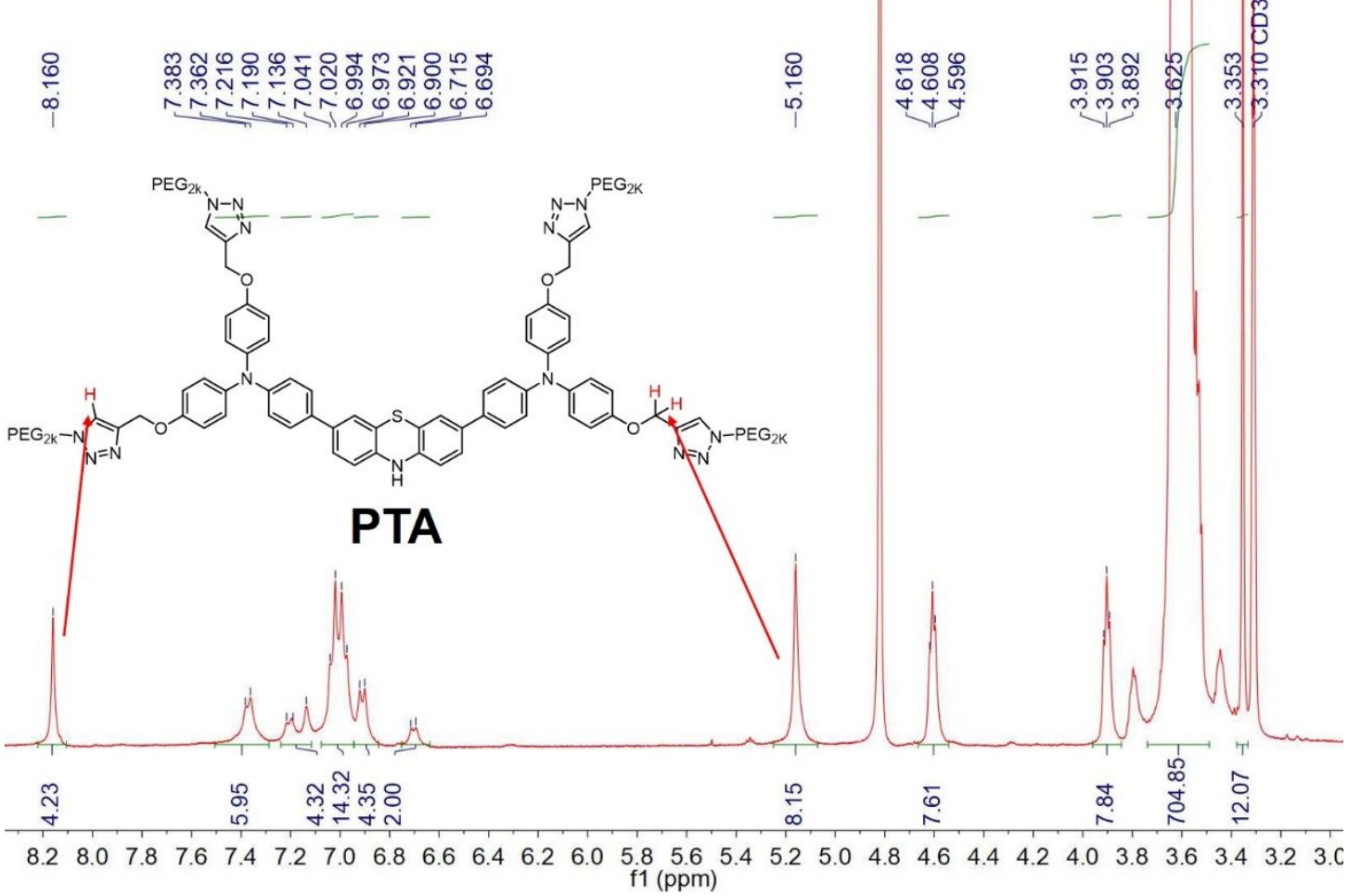




\section{LC-MS data of core structures in PTA and PTA-CIO}
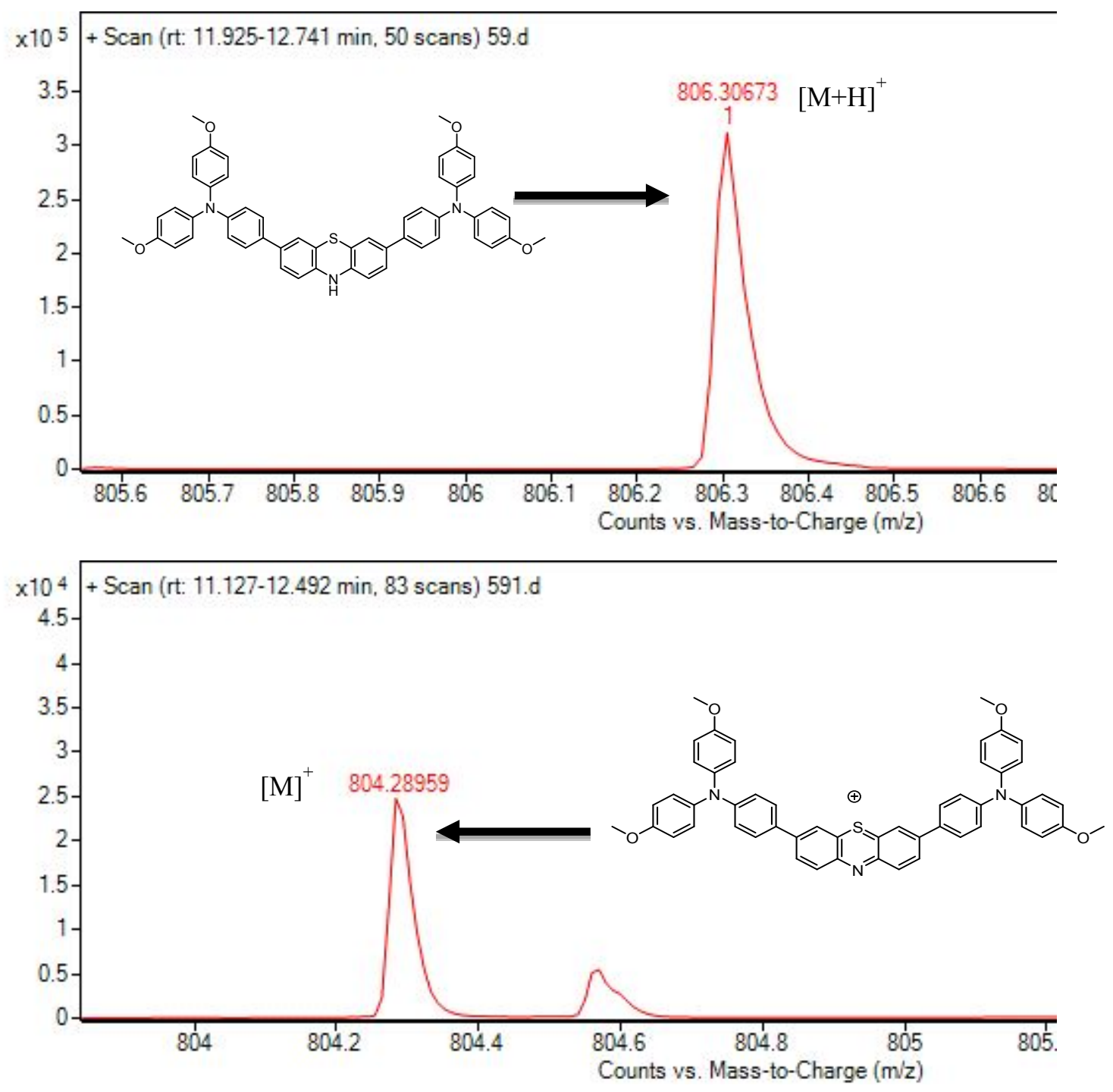


\section{References}

(1) Best, Q. A.; Sattenapally, N.; Dyer, D. J.; Scott, C. N.; McCarroll, M. E. J. Am. Chem. Soc. 2013, 135, 13365-13370.

(2) Koide, Y.; Urano, Y.; Hanaoka, K.; Terai, T.; Nagano, T. J. Am. Chem. Soc. 2011, 133, 5680-5682.

(3) Wei, P.; Yuan, W.; Xue, F.; Zhou, W.; Li, R.; Zhang, D.; Yi, T. Chem. Sci. 2018, 9, 495-501.

(4) Xiao, H.; Xin, K.; Dou, H.; Yin, G.; Quan, Y.; Wang, R. Chem. Commun. 2015, 51, 1442-1445.

(5) Cheng, G.; Fan, J.; Sun, W.; Cao, J.; Hu, C.; Peng, X. Chem. Commun. 2014, 50, 1018-1020.

(6) Zhang, W.; Liu, W.; Li, P.; kang, J.; Wang, J.; Wang, H.; Tang, B. Chem. Commun. 2015, 51, 10150-10153.

(7) Hou, J. T.; Li, K.; Yang, J.; Yu, K. K.; Liao, Y. X.; Ran, Y. Z.; Liu, Y. H.; Zhou, X. D.; Yu, X. Q. Chem. Commun. 2015, 51, 6781-6784.

(8) Long, L.; Zhang, D.; Li, X.; Zhang, J.; Zhang, C.; Zhou, L. Anal. Chim. Acta 2013, 775, 100-105.

(9) Emrullahoglu, M.; Ucuncu, M.; Karakus, E. Chem. Commun. 2013, 49, 7836-7838.

(10) Cheng, G.; Fan, J.; Sun, W.; Sui, K.; Jin, X.; Wang, J.; Peng, X. Analyst 2013, 138, 6091-6096.

(11) Wu, W.-L.; Zhao, Z.-M.; Dai, X.; Su, L.; Zhao, B.-X. Sens. Actuators B Chem. 2016, 232, 390395.

(12) Goswami, S.; Maity, S.; Maity, A. C.; Das, A. K. Sens. Actuators B Chem. 2014, 204, 741-745.

(13) Xiao, H.; Li, J.; Zhao, J.; Yin, G.; Quan, Y.; Wang, J.; Wang, R. J. Mater .Chem. B 2015, 3, $1633-$ 1638.

(14) Zhang, X.; Zhao, W.; Li, B.; Li, W.; Zhang, C.; Hou, X.; Jiang, J.; Dong, Y. Chem. Sci. 2018, 9, 8207-8212.

(15) Zhang, W.; Guo, C.; Liu, L.; Qin, J.; Yang, C. Org. Biomol. Chem. 2011, 9, 5560-5563. 